\title{
A Vaginal Cyst Causing Dysuria and Dyschezia in a Bitch
}

\author{
Toshifumi WATANABE ${ }^{1) *}$, Mika MISHINA $^{1)}$ and Yohei SAKURAI ${ }^{1)}$ \\ 1)Department of Nephrology-Urology, Azabu University Veterinary Teaching Hospital, 1-17-71 Fuchinobe, Chuo-ku, Sagamihara-shi, \\ Kanagawa 252-5201, Japan
}

(Received 25 September 2013/Accepted 5 March 2014/Published online in J-STAGE 20 March 2014)

ABSTRACT. A 9-year-old female dog was referred to Azabu University Veterinary Teaching Hospital for dysuria and dyschezia after ovariohysterectomy. After a series of diagnostic imaging, vaginal obstruction was suspected by vaginal fluid retention. Surgical repair was attempted three times to establish patency through the vagina and the vestibule by episiotomy and laparotomy. Another laparotomy was performed to remove the entire vaginal mucosal layer to prevent recurrence, which resulted in favorable outcome. Histopathological examination revealed that the resected tissue was a cyst originated from mesonephric duct remnant. In the present case, the cyst was curable by the entire resection of the cyst lining membrane, which could eradicate all the secretory cells with least damage to the urethral vasculature and innervation.

KEY WORDS: canine, dysuria, vaginal cyst.

doi: 10.1292/jvms.13-0471; J. Vet. Med. Sci. 76(7): 1039-1043, 2014

A vaginal cyst is a formation of cystic structure within the vaginal wall. It may arise from a remnant of the embryonic urogenital duct or an entrapment of the mucosal epithelium under the mucosal layer by trauma or surgery $[1,2,8]$. Vaginal cysts are classified based on the type of epithelial cells that are lining the cyst, such as Müllerian cysts, Gartner's ducts cysts and epithelial inclusion cysts [2, 8]. In humans, the majority of vaginal cysts are asymptomatic, however, which may cause pain, dysuria and/or dystocia due to their enlargement. Therefore, surgical intervention, such as cystectomy and marsupialization, is required $[2,8]$. In veterinary medicine, vaginal cysts are known to be one of common reproductive failure in cattle [3], which are rarely seen in dogs $[1,5]$. As in humans, canine vaginal cysts are either asymptomatic or symptomatic depending on the size [1], and surgical repair is required for symptomatic cysts [5]. Laparotomic cystectomy can be applied to remove the cysts [5], however, one study reports that marsupialization is preferred to keep the urinal vasculature and innervation intact [4]. In the present study, we report a case of a vaginal cyst observed in a dog experiencing dysuria and dyschezia, which was successfully controlled by entire resection of the cyst lining membrane.

A 9-year-old, female Shih Tzu dog, weighing $3.74 \mathrm{~kg}$ (body condition score of 2/5), was presented to a referral veterinary clinic for neutering. Ovariohysterectomy was performed, and then, the uterine cervix was found enlarged in a ball-like shape, $5 \mathrm{~cm}$ in diameter. The enlarged uterine cervix was removed at its most caudal end with the ovaries and

*Correspondence to: Watanabe, T., Azabu University Veterinary Teaching Hospital, 1-17-71 Fuchinobe, Chuo-ku, Sagamihara-shi, Kanagawa 252-5201, Japan. e-mail: watanabe@azabu-u.ac.jp (C2014 The Japanese Society of Veterinary Science

This is an open-access article distributed under the terms of the Creative Commons Attribution Non-Commercial No Derivatives (by-nc-nd) License $<$ http://creativecommons.org/licenses/by-nc-nd/3.0/>. uterine horns. It was filled with brown-colored and viscous sterile fluid, and the cervical lining membrane was smooth without any evidence of mass. The ovaries and uterus horns were not submitted for histopathological examination. The dog developed dysuria on the following day of the ovariohysterectomy and was followed by dyschezia two weeks later. A $6 \mathrm{~cm} \times 3 \mathrm{~cm}$ non-echogenic mass was detected caudal to the bladder by abdominal ultrasonography, and then, the dog was referred to Azabu University Veterinary Teaching Hospital for detailed evaluation and treatment (Day 0).

During physical examination, a 5-cm mass was palpated caudal to the bladder. Results of CBC and serum chemistry were within reference range. By survey radiography, the soft tissue density mass was detected caudal to the bladder, and the colon was displaced dorsally due to its presence (Fig. 1A). Abdominal ultrasonography revealed a non-echogenic fluid-filled structure (Fig. 2). The aspirated fluid was brown-colored and viscous, and neutrophils and nucleated cornified epithelial cells were also detected by cytology. The fluid was negative for bacterial culture. Vaginourethrography was then performed, contrast medium did not fill the vagina, and ventral displacement of the urethra was also evident (Fig. 1B). Based on these findings, we suspected that dysuria and dyschezia were caused by vaginal obstruction, which led to the vaginal fluid retention and mechanical compression of the urethra and the colon.

On Day 2, an episiotomy was performed to examine the cause of the vaginal obstruction and to drain the cyst under anesthesia. After pretreatment with $0.025 \mathrm{mg} / \mathrm{kg}$ atropine sulfate (ATROPINE SULFATE Injection $0.5 \mathrm{mg}$, Mitsubishi Tanabe Pharma, Osaka, Japan), SC, and anesthetic induction with $6 \mathrm{mg} / \mathrm{kg}$ propofol (1\% Propofol Injection, Mylan Seiyaku, Tokyo, Japan), IV, the patient was intubated and maintained on isoflurane (ISOFLU ${ }^{\circledR}$, DS Pharma Animal Health, Osaka, Japan). Antibiotic, cefovecin sodium $8 \mathrm{mg} / \mathrm{kg}$ (CONVENIA ${ }^{\circledR}$, Pfizer, Tokyo, Japan), was administered subcutaneously before the surgical examina- 

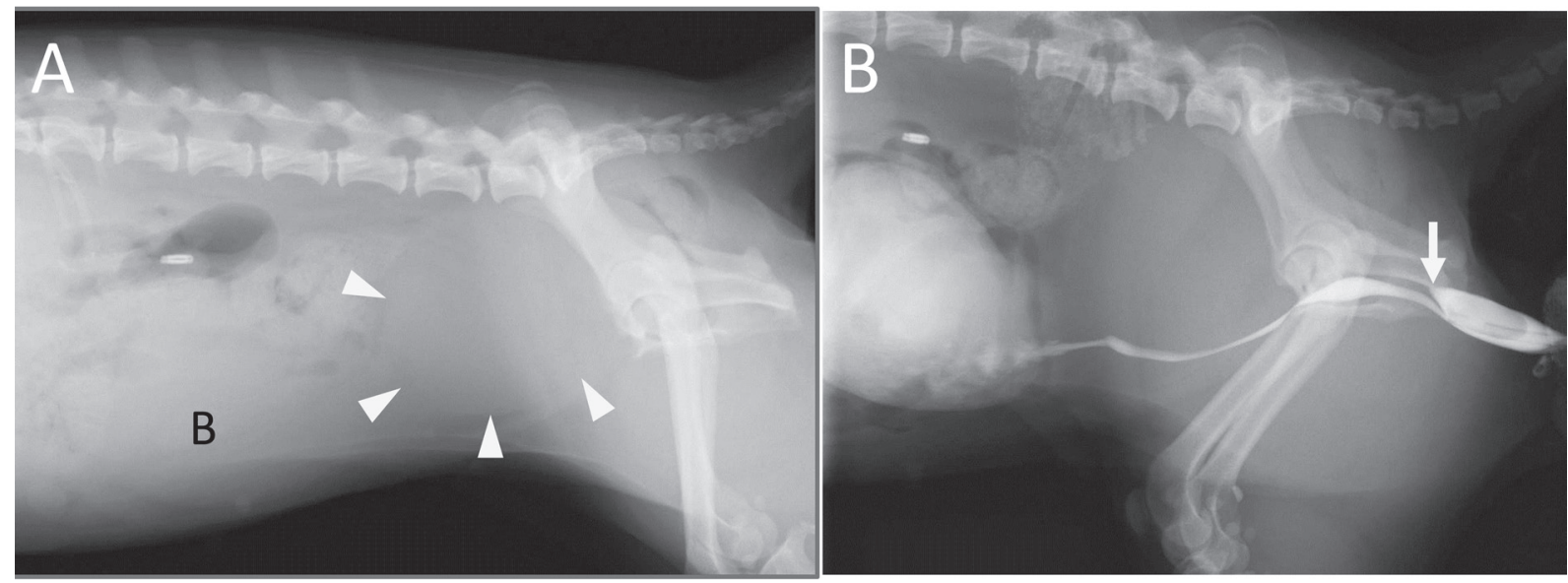

Fig. 1. (A) A lateral radiograph of the abdomen. The soft tissue density mass (arrowhead) was observed caudal to bladder (B), and the colon was displaced dorsally due to the presence of the mass. (B) A lateral vaginourethrogram showing a contrast filling defect in the vagina. The contrast medium stopped at the cranial end of the vestibule (arrow), indicating blind ending of the vestibule. The urethra was displaced ventrally due to the presence of the mass.

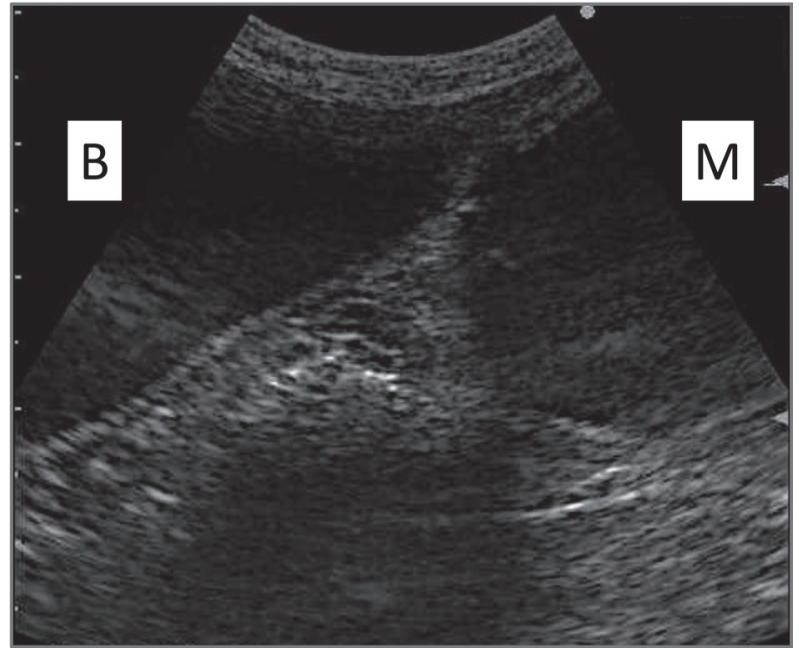

Fig. 2. Caudal abdominal ultrasonography. There was a nonechogenic fluid-filled mass (M) caudal to the bladder (B).

tion. The patient was positioned in ventral recumbency with the pelvic limbs hanged over the edge of the table. After an incision in the perineum, it was visually confirmed that the vestibule was blind ending cranially to the external urethral orifice (Fig. 3A). The smooth and blind end was cut open and removed with forceps, the cranial part was incised with a scalpel, and the fluid ran out of the vagina. The fluid was withdrawn, and the incision was extended to visualize the vaginal mucosa. Marsupialization was then performed by suturing the vaginal mucosa and the incised vestibular mucosa (Fig. 3B).

Postoperatively, there was an improvement in urination and defication. However, on Day 7, dysuria and dyschezia developed again, and recurrence of vaginal fluid retention was detected by the abdominal ultrasonography. Therefore, an exploratory laparotomy was performed on Day 9 to further investigate the cause of fluid retention, pretreatment and anesthesia as previously explained. The patient was positioned in dorsal recumbency, and the abdominal cavity was exposed by a median incision in the lower abdomen. It was found that a distended vagina was severely adhered to the bladder neck and the urethra (Fig. 4). The bladder was inverted to examine the cranial part of the vagina, but there was no evident sign of stump pyometra, cysts or any other abnormalities that could cause the vaginal fluid retention. The bladder was returned to its original position, and a further 2-cm incision was made in the ventral wall of the vagina to place drainage. Forceps were inserted through the incision and advanced caudally to re-establish the patency through the vagina and the vestibule. An 8-French balloon catheter (All Silicone Foley Balloon Catheter, Create Medic, Yokohama, Japan) was inserted from the vulva by guiding its tip with forceps and placed in the vagina. The vaginal incision was sutured with 3-0 polyglyconate, and the laparotomy was closed in a standard manner.

Urination and defecation improved postoperatively. Escherichia coli was detected by bacterial culture, using the vaginal fluid and urine samples which were obtained during the operation. Based on drug susceptibility test, $5 \mathrm{mg} / \mathrm{kg}$ imipenem hydrate and cilastatin sodium (TIENAM ${ }^{\circledR}$ for Intravenous Drip Infusion 0.25 g, MSD, Tokyo, Japan) was administered intravenously after the surgery. During hospitalization, the vagina was washed with saline using a balloon catheter twice a day. The amount of vaginal fluid gradually decreased. On Day 16, only a minimal amount of vaginal fluid was detected by abdominal ultrasonography, so the patient was discharged after removing the catheter on the same day. However, on Day 28, dysuria and dyschezia recurred. Abdominal ultrasonography revealed an enlarged vagina to the size of $6 \mathrm{~cm} \times 3 \mathrm{~cm}$. An attempt to insert a 

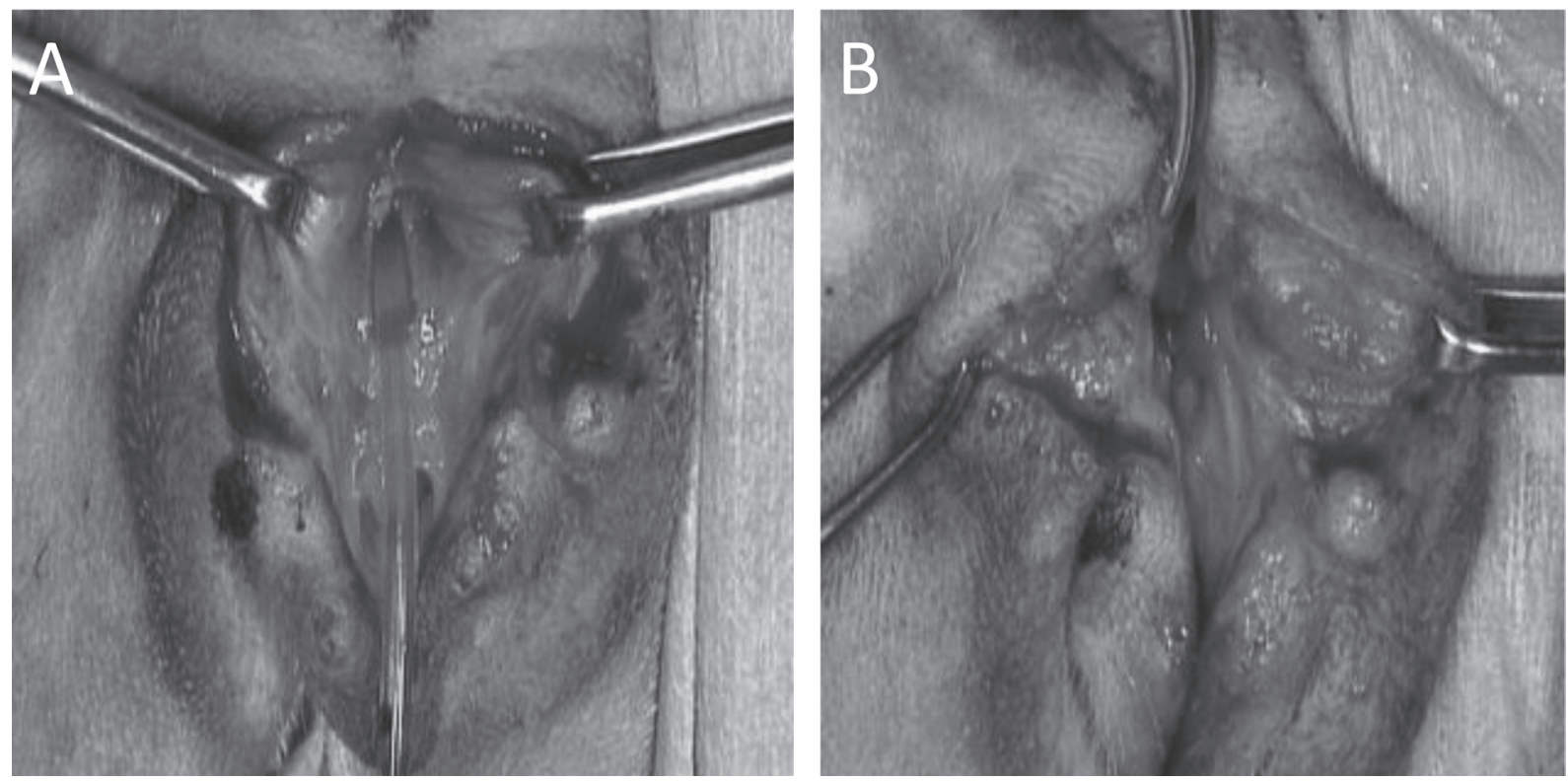

Fig. 3. Images at the first surgery. (A) The vaginal vestibule was blind ending cranially to the external urethral orifice. (B) Marsupialization was performed.

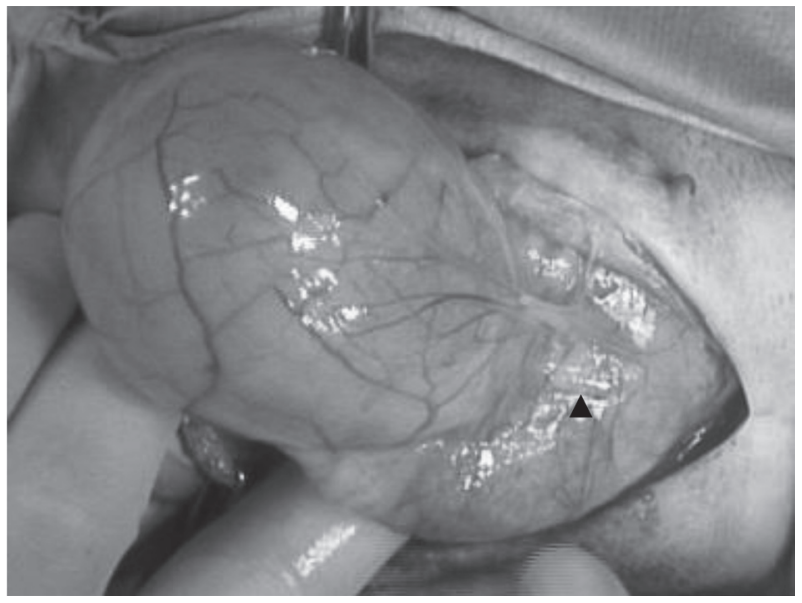

Fig. 4. An image at the second surgery. The distended vagina was severely adhered to the bladder neck and urethra (arrowhead).

catheter through the vulva into the vagina was unsuccessful, indicating that due to failure of the re-establishment of patency, another vaginotomy was performed on Day 37.

After performing the anesthetic procedure as previously explained, the patient was positioned in ventral recumbency. When the vestibule was exposed by episiotomy, it was evident that the marsupialized opening had been fully closed. The closure was incised, and forceps were advanced cranially to separate the tissue. A membranous structure was visually confirmed deeper in the vagina (Fig. 5A). The patient was repositioned in dorsal recumbency for a lower median laparotomy to re-establish the patency, since the incision was unable to perform due to its thickness. Similar to the second surgery, a 2-cm incision was made in the ventral wall of the vagina, and forceps were advanced caudally, however, the vestibule was not reachable at this time. The incision was extended cranially and caudally to expose the vaginal mucosa, and the entire mucosal layer was separated from the serosa and fully removed (Fig. 5B). The serous membrane was not sutured, and the abdomen was closed in the standard manner.

Histopathological examination of the resected tissue with H\&E staining revealed a lack of the stratified squamous epithelial lining, which is normally seen in the vaginal mucosa. Instead, the tissue was lined with simple cuboidal and columnar epithelia (Fig. 6A). Moreover, under the mucosal layer, some plasma cells, lymphocytes and macrophages as well as glandular structures were observed (Fig. 6B). From these findings, chronic active inflammation was diagnosed in the cystic structure, and the site of cyst formation suggested that the resected tissue may be a remnant of a duplicated bladder, Gartner's duct or some other remnant structure originated from the mesonephric duct.

After the third surgery, no recurrence of the cyst was detected. Although the patient experienced incontinence while sleeping, the symptom gradually subsided and resolved after six months by administration of clenbuterol hydrochloride $10 \mu \mathrm{g} /$ head (SPIROPENT ${ }^{\circledR}$ Tablet $10 \mu \mathrm{g}$, Teijin Pharma, Tokyo, Japan), PO, bid. After the surgery, this patient remained stable with no further problems regarding to urination and defication.

In the present case, a Gartner's duct cyst was indicated as the main cause of dysuria and dyschezia. During the embryonic development, the mesonephric duct differentiates into the deferent duct in males. It normally regresses in female 

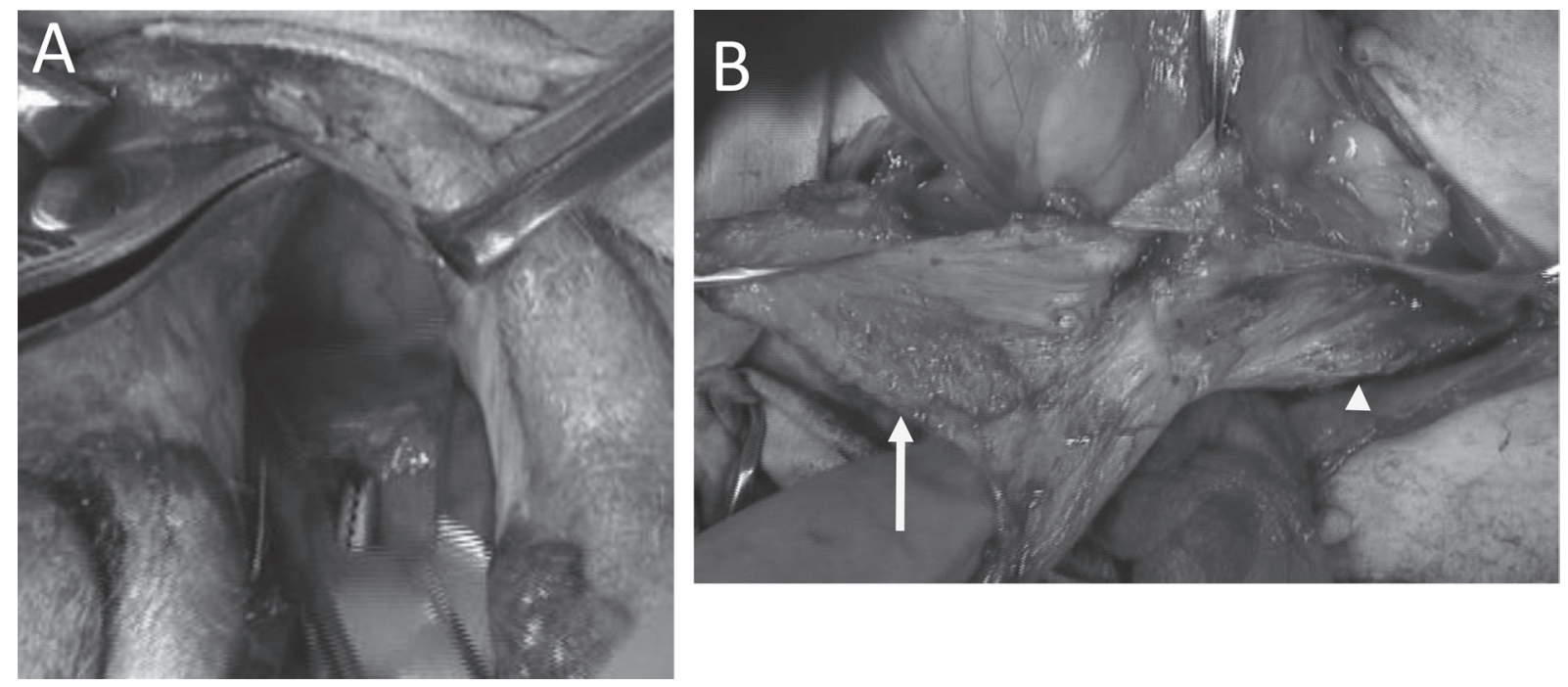

Fig. 5. Images at the third surgery. (A) A membrane-like structure was present in the cranial vagina. (B) The cyst lining (arrow) was separated from the serous membrane (arrowhead).
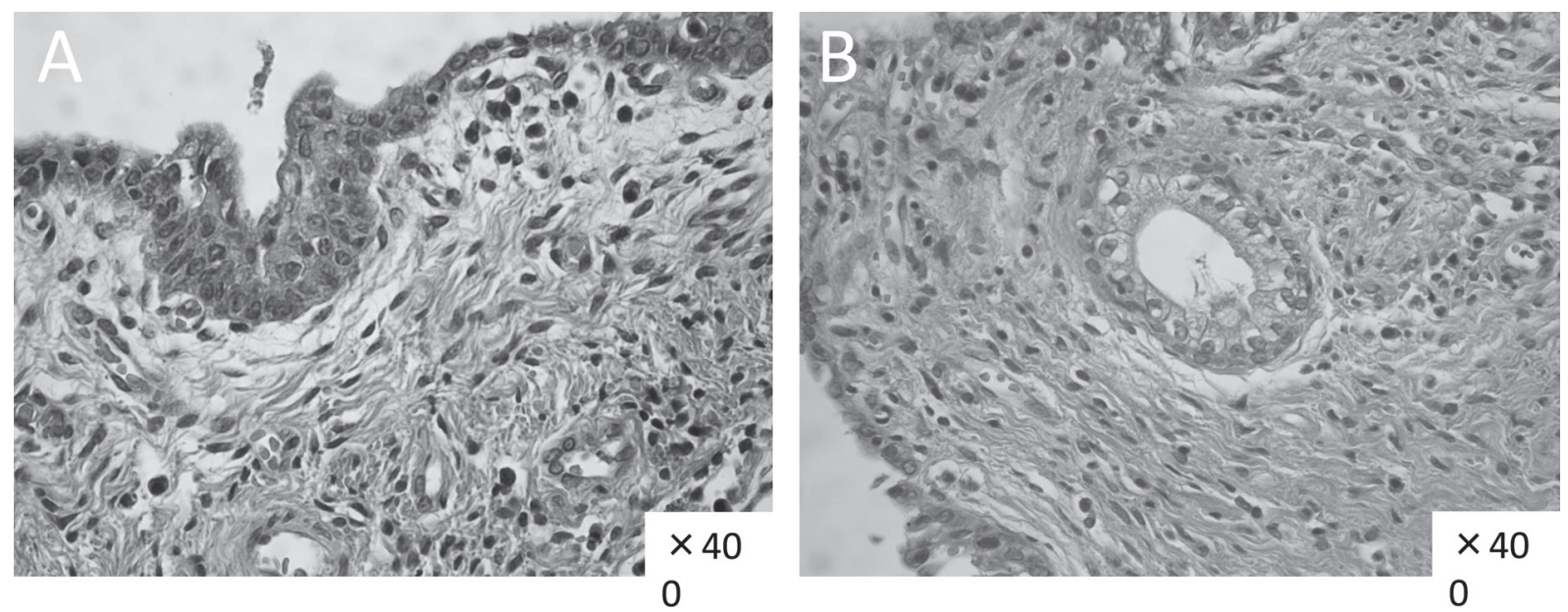

Fig. 6. Histopathological images of the cyst. H\&E staining, $\times 400$. (A) The cyst was lined with simple cuboidal and columnar epithelial cells. (B) A glandular structure was present under the mucosal layer.

mammals, however, the caudal part may persist as a Gartner's duct in the vaginal wall $[6,7]$. A Gartner's duct cyst is formed as a result of secretion from the glandular cells present in the Gartner's duct [5, 8]. As the secretions accumulated by the inflammation of the cyst caused by surgical invasion, such as ovariohysterectomy, the cyst may enlarge and cause mechanical interference with other organs, such as the urethra and the colon, and this may result in dysuria and dyschezia as seen in the present case.

In the present case, since vaginal obstruction was strongly suspected by the first vaginourethrography and intraoperative findings at the first surgery, the presence of a Gartner's duct cyst was not diagnosed. Clinical symptoms and findings, such as pain, dysuria and dyschezia, may develop in both vaginal obstruction and Gartner's duct cysts due to physical urethral and/or intestinal compression $[9,10]$. Imaging findings are also similar, both typically being detected as a mass-like opacity caudal to the bladder by radiographs, and a non-echogenic fluid-filled structure is detected by ultrasonography. On the other hand, vaginography may play a key role to differentiate vaginal obstruction from Gartner's duct cyst. A contrast-filled defect in the vagina is typical for vaginal obstruction, while with a Gartner's duct cyst, the vaginal part caudal to the cyst should be easily seen. In the present case, the vagina was not filled with the contrast medium, which leads to difficulty in making a diagnosis of vaginal cyst (Fig. 7).

Marsupialization has been recommended for surgical repair of vaginal cysts by Holt [4]. Although it is known to be the least invasive technique, it is not suitable for small- 


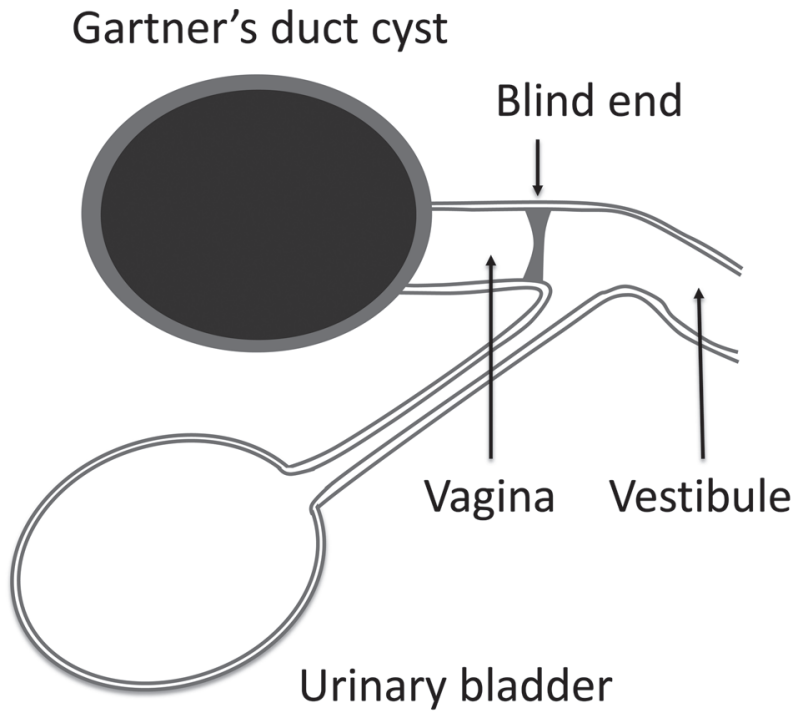

Fig. 7. A schematic image of the Gartner's duct cyst observed in the present case.

breed dogs and cysts which are located to the cranial part of the vagina. This can be explained that suturing the cyst wall and vaginal mucosa would be technically difficult due to the limited operative field. The glandular cells in the cysts may continue to secrete mucosal fluid even after the surgery. Thus, if the marsupialized opening is fully closed, there exists a risk of recurrence. Cystectomy would be curative surgical method, but if there is a severe adhesion with the urethra, colon and/or other surrounding structures, urethral blood vessels and nerves may be damaged during intraoperative manipulation of a cyst that is located caudal to the urethra [4].

In the present case, since marsupialization and cystectomy were difficult, only the cyst lining membrane was removed leaving the serous membrane. However, recurrence of cyst distention has not been observed. Although transient incontinence was experienced postoperatively due to surgi- cal invasion, the patient was able to urinate and defecate without any problems at Day 650. We attribute this successful outcome to the complete eradication of glandular cells in the cyst lining membrane, as well as the minimally invasive approach to preserve the surrounding tissues without damaging the urethral vasculature and innervation. Thus, removal of the cyst lining membrane may be a noble and effective option for symptomatic vaginal cysts with adhesions to the surrounding structures.

\section{REFERENCES}

1. Cauvin, A., Sullivan, M., Harvey, M. J. and Thompson, H. 1995. Vaginal cysts causing tenesmus in a bitch. J. Small Anim. Pract. 36: 321-324. [Medline] [CrossRef]

2. Crum,C.P., Nicci,M.R. and Lee,K.R. 2011: pp. 215-216. Diagnostic Gynecologic and Obstetric Pathology, 2nd ed., Saunders, Philadelphia.

3. Ginther, O. J. 1965. Segmental aplasia of the Mullerian ducts (white heifer disease) in a White Shorthorn heifer. J. Am. Vet. Med. Assoc. 146: 133-137. [Medline]

4. Holt, P. E. 1993. Urinary retention in a bitch. Vet. Rec. 132: 592. [Medline] [CrossRef]

5. Kim, H. J., Kim, J. K., Choi, J. H., Jang, J. Y., Ban, H. J., Seo, J. M., Lee, M. J., Choi, H. Y., Kim, M. K. and Kim, H. W. 2007. A Gartner duct cyst of the vagina causing dysuria and dyschezia in a Yorkshire Terrier. J. Vet. Sci. 8: 427-429. [Medline] [CrossRef]

6. Mcgeady, T. A., Quinn, P. J., FitzPatrick, E. S. and Ryan, M. T. 2006. Genital ducts. pp. 249-251. In: Veterinary Embryology. Wiley-Blackwell, Hoboken.

7. Moore, K. L., Persaud, T. V. N. and Torchia, M. G. 2013. Development of Genital Ducts. pp. 269-273. In: The Developing Human, 9th ed., Saunders, Philadelphia.

8. Ostrzenski,A. 2002: pp. 220-221. Gynecology: Integrating Conventional, Complementary, and Natural Alternative Therapy. Lippincott Williams \& Wilkins, Philadelphia.

9. Tsumagari, S., Takagi, K., Takeishi, M. and Memon, M. A. 2001. A case of a bitch with imperforate hymen and hydrocolpos. $J$. Vet. Med. Sci. 63: 475-477. [Medline] [CrossRef]

10. Viehoff, F. W. and Sjollema, B. E. 2003. Hydrocolpos in dog: surgical treatment in two cases. J. Small Anim. Pract. 44: 404-407. [Medline] [CrossRef] 